\title{
基于非参数估计的双模态红外图像差异特征频次分布构造
}

\author{
张雅玲，吉琳娜，杨风暴，母晓慧 \\ (中北大学 信息与通信工程学院, 山西 太原 030051)
}

\begin{abstract}
摘要: 差异特征频次属性的分布构造对建立双模态红外图像差异特征多属性融合有效度分布合成具有 重要意义。针对双模态红外图像差异特征频次属性分布构造的问题, 提出了基于 $K$ 最近邻 $\left(K_{\mathrm{NN}}\right)$ 概 率密度估计的差异特征频次分布构造方法。利用累积分布函数得到差异特征频次真实序列值, 计算所 构造的差异特征频次分布中具有统计意义的频次序列值与真实序列值的相似性测度, 对结果进行了验 证。实验结果表明，将非参数概率密度估计运用于差异特征频次分布构造中具有可行性，且本文方法 相较于 MISE 最优带宽高斯核密度估计更能准确构造差异特征频次分布。
\end{abstract}

关键词: 非参数概率密度估计; 差异特征频次; 复化梯形积分; 相似性测度

中图分类号：TP391 文献标识码：A 文章编号：1001-8891(2020)04-0361-09

\section{Bimodal Infrared Images of Frequency Distribution of Difference Features Based on Nonparametric Estimation}

\author{
ZHANG Yaling, JI Linna, YANG Fengbao, MU Xiaohui \\ (Information and Communications Engineering College, North University of China, Taiyuan 030051, China)
}

\begin{abstract}
The distribution of difference feature frequency is crucial for establishing a multi-attribute fusion validity distribution synthesis of difference features of bimodal infrared images. To construct different feature frequency distributions of bimodal infrared images, a method of constructing a difference feature frequency distribution based on the $K$ nearest neighbor $\left(K_{\mathrm{NN}}\right)$ probability density estimation is proposed. The cumulative distribution function is used to obtain the true sequence value of the difference feature frequency; subsequently, the similarity measure of the statistically significant frequency sequence value and the real sequence value in the constructed frequency distribution of the difference feature are calculated. Experimental results show that non-parametric probability density estimation can be applied to the frequency distribution of difference features. The proposed method can accurately construct the frequency distribution of difference features compared with the MISE optimal bandwidth Gaussian kernel density estimation.
\end{abstract}

Key words: nonparametric probability density estimation, difference feature frequency, complex trapezoidal integral, similarity measure

\section{0 引言}

双模态红外图像存在大气传输、成像仪响应以及 目标与背景辐射强度等差异性, 导致这两类图像所呈 现特征的差异很大 ${ }^{[1]}$ 。红外光强图像主要基于物体红外 热辐射强度成像, 该类图像的亮度信息较为明显, 纹 理及边缘信息较少; 红外偏振度图像主要基于物体多 方向偏振辐射量成像 ${ }^{[2]}$, 该类图像中目标的细节丰富且 边缘清晰, 亮度信息较少。
双模态红外图像的差异特征具有类型、幅值和频 次等属性。差异特征频次属性从宏观上讲, 反映了成 像场景中某种差异特征分布范围的广泛性; 从微观上 讲, 反映了特定的差异特征随着差异特征幅值变化在 成像场景中分布的疏密程度。文献[3]构建了图像组中 每个图像块各类型差异特征幅值对应融合算法的融合 有效度分布, 利用数据包络分析法 (data envelopment analysis, DEA）建立差异特征与融合算法的集值映射, 文献[4]研究了同类和异类差异特征幅值融合有效度的 
分布合成, 验证了融合有效度分布合成的有效性, 上 述两篇文献考虑了基于类型和幅值的差异特征融合有 效度分布的构建和合成, 但忽视了差异特征频次属性 对融合的影响, 最终的结果会产生偏差。目前对于差 异特征的研究只针对类型和幅值这两种属性, 当差异 特征频次大小发生变化时, 融合算法及融合规则的选 取也会随之变化。现有的这两种属性无法有效地反映 出差异特征多属性对融合算法选取的影响, 导致融合 效果差甚至融合方法失效, 所以差异特征频次的研究 在图像融合中起着十分关键的作用。

因为差异特征类型、幅值分布疏密程度等因素与 频次分布的函数关系是未知的, 所以不同类型、不同 幅值分布下无法准确得出差异特征频次服从哪些具 体分布。不同差异特征幅值的分布能反映出差异特征 频次的变化, 差异特征幅值的概率密度分布又可以通 过参数估计法得到。参数估计法中的非参数估计法针 对的分布函数具体形式是未知的, 样本函数与其概率 密度函数服从同分布 ${ }^{[5]}$, 由于差异特征幅值函数形式 是未知的, 所以本文采用非参数概率密度估计的方法 可以得到差异特征幅值样本集的概率密度分布, 从而 构造出差异特征频次分布。另外两种参数估计方法全 参数估计和半参数估计均需要在分布函数具体形式 已知的情况下进行估计 ${ }^{[5]}$, 故不符合本文的要求。常 用的非参数概率密度估计法有 $K_{N \mathrm{~N}}$ 概率密度估计和核 密度估计法。本文所提出的基于 $K_{N \mathrm{~N}}$ 概率密度估计的 差异特征频次分布构造法和核密度估计相比, 避免了 核密度概率密度估计中核函数以及核函数带宽选择 的主观性, 因此能准确构造差异特征频次分布。

\section{1 背景理论}

双模态红外图像是指分别基于红外热辐射成像 和红外偏振成像这两种不同的红外探测机理, 并对一 定的目标场景进行图像采集最后经过相应的图像信 息解算所得到的两种模态的红外图像, 亦即红外光强 图像和红外偏振度图像。偏振光相较于传统的红外线 可以将光的多个偏振形态表示出来, 进而将许多人眼 无法觉察到的信息显示出来, 便于目标探测与识别。 斯托克斯表示法 ${ }^{[6]}$ 是常见的光波强度和偏振态的描述 法, 它利用 4 个参数 $I 、 Q 、 U 、 V$ 可以将光的偏振状 态全部表示出来, 这 4 个参数称为斯托克斯参数, 可 以直接测量。设准偏振光沿 $Z$ 方向传播, 其平均频率 为 $v$, 并设电矢量 $E$ 的 $x 、 y$ 分量分别为 $E_{x} 、 E_{y}$, 如公 式(1)所示:

$$
\begin{aligned}
& E_{x}(t)=a_{x}(t) \cos \left[\varphi_{1}(t)-2 \pi v t\right] \\
& E_{y}(t)=a_{y}(t) \cos \left[\varphi_{2}(t)-2 \pi v t\right]
\end{aligned}
$$

式中: $a_{x}(t)$ 和 $a_{y}(t)$ 分别为电矢量 $E$ 的振幅, 化解掉 公式(1)中的 $-2 \pi v t$, 如公式(2)所示:

$$
\frac{E_{x}^{2}(t)}{a_{x}^{2}(t)}+\frac{E_{y}^{2}(t)}{a_{y}^{2}(t)}-2 \frac{E_{x}(t)}{a_{x}(t)} \frac{E_{y}(t)}{a_{y}(t)} \cos \delta(t)=\sin ^{2} \delta(t)
$$

式中: $\delta(t)=\varphi_{2}(t)-\varphi_{1}(t)$ 是 $E_{x} 、 E_{y}$ 的相位差。

经过复杂的公式推导, 由于偏振光的振幅和相位 差与时间相关, 可以得到推广式 ${ }^{[7]}$, 如公式(3)所示:

$$
\left\{\begin{array}{l}
S_{0}=I=\left\langle a_{x}{ }^{2}(t)\right\rangle+\left\langle a_{y}{ }^{2}(t)\right\rangle \\
S_{1}=Q=\left\langle a_{x}^{2}(t)\right\rangle-\left\langle a_{y}{ }^{2}(t)\right\rangle \\
S_{2}=U=2\left\langle a_{x}(t) a_{y}(t) \cos \delta(t)\right\rangle \\
S_{3}=V=2\left\langle a_{x}(t) a_{y}(t) \sin \delta(t)\right\rangle
\end{array}\right.
$$

式中: $I$ 表示光的总强度; $Q$ 表示 $0^{\circ}$ 方向和 $90^{\circ}$ 方向线 偏振光分量强度差值; $U$ 表示 $45^{\circ}$ 与 $135^{\circ}$ 方向线偏振 光分量强度差值; 而 $V$ 代表右旋与左旋圆偏振光分量 之差。因为在实际探测过程中, 圆偏振光的分量极少 且相较于仪器误差是可以忽略不计的, 通常认为 $V=$ 0 , 因此可以直接利用 $I 、 Q 、 V$ 三个独立的斯托克斯 参数来准确表示一束光的偏振态, 当一束光源与水平 轴 $x$ 夹角为 $\alpha$ 时, 观测到的光强度如公式(4)所示:

$$
I(\alpha)=\frac{1}{2}(I+Q \cos 2 \alpha+U \sin 2 \alpha)
$$

本文将 $0^{\circ}$ 设为参考方向并确立为初始位置, 将偏 振片逐步旋转到 $45^{\circ} 、 90^{\circ} 、 135^{\circ}$ 三个不同位置, 并将 这 4 个不同偏振方向的光强分量代入到公式(4)中, 化 解可得:

$$
\left\{\begin{array}{l}
I=I_{0^{\circ}}+I_{90^{\circ}}=I_{+45^{\circ}}+I_{-45^{\circ}}=I_{1}+I_{\mathrm{r}} \\
Q=I_{0^{\circ}}+I_{90^{\circ}} \\
U=I_{+45^{\circ}}-I_{-45^{\circ}} \\
V=I_{\mathrm{r}}-I_{1}
\end{array}\right.
$$

式中: $I_{0^{\circ}} 、 I_{90^{\circ}} 、 I_{+45^{\circ}} 、 I_{-45^{\circ}} 、 I_{\mathrm{r}} 、 I_{1}$ 分别表示放置在光 波传播路径上的理想偏振片在 $0^{\circ} 、 90^{\circ} 、+45^{\circ} 、-45^{\circ}$ 方向上的线偏振光以及左旋 1 和右旋 $\mathrm{r}$ 圆偏振光。通 过测出 3 个不同角度的光强分量可以直接计算出斯托 克斯参数 ${ }^{[8]}$, 在图像中, 参数 $I$ 代表了物体的强度信 息, 即反映不同物体的反射比。参数 $Q$ 代表了物体的 材质, 参数 $U$ 代表了边缘和轮廓信息。偏振光的线偏 振度 $P$ 和偏振角 $\theta$ 如公式(6)所示: 


$$
\left\{\begin{array}{l}
P=\frac{\sqrt{Q^{2}+U^{2}}}{I} \\
\alpha=\frac{1}{2} \arctan \left(\frac{U}{Q}\right)
\end{array}\right.
$$

本红外偏振探测系统选取偏振度图像作为偏振 成像的单模态图像, 偏振度图像能够体现物体的表面 边缘信息, 是从自然背景中凸显出人造目标特征的方 法 $^{[9]}$, 红外偏振度图像可以很好地表示出物体材质、 粗糙特性、边缘特征、轮廓信息、纹理细节, 目标的 对比反差度特性, 但偏振片得到的辐射能量被削弱, 红外辐射强度很低, 所以红外偏振度图像的光强信息 很弱。红外热辐射成像由于直接对目标物体进行热辐 射强度成像, 并未对光的多个方向的偏振态进行成 像, 所以得到的红外图像光强信息很高, 但物体材质、 纹理边缘细节、对比度信息很弱。由于红外偏振度图 像和红外光强图像这两个单模态图像成像特性差异 巨大，所以二者组成的双模态图像具有极大的互补性 信息。通常情况下, 用辐射对比度和偏振度对比度分 别描述目标和背景的热辐射强度差异和偏振特性差 异, 辐射对比度对应在图像特征信息中表示亮度, 而 偏振度对比度对应在图像特征信息中表示边缘幅值 强度、反差度、边缘清晰度、粗粮特性和对比度等。

差异特征幅值是指双模态红外图像特征值间强 度的绝对差异度, 如公式(7)所示:

$$
T=\left|T_{\mathrm{I}}-T_{\mathrm{P}}\right|
$$

式中: $T_{\mathrm{I}} 、 T_{\mathrm{P}} 、 T$ 分别表示红外偏振度图像、光强图 像以及两类图像对应图像块特征值差值的强度。在不 同的成像场景中, 两类图像在对应的不同局部图像块 中辐射对比度差异值或者偏振度对比度差异值都不 同, 但存在部分辐射对比度差异值或偏振度对比度差 异值的大小近似相同, 在图像特征信息中可表示为两 类图像中亮度特征、边缘幅值强度、反差度、边缘清 晰度、粗粘度和对比度等差异特征幅值大小不同, 但 存在大小相同的差异特征幅值 (本文中将幅值大小完 全相同和在一定的小范围幅值区间内的近似相同均 视为相同)。

统计并研究不同大小的辐射对比度差异值和偏 振度对比度差异值出现的频次能够深刻地反映出成 像中热辐射强度信息和偏振度信息在双模态红外图 像中分布规律, 同时对于提高双模态红外图像融合质 量提供了思路, 上述两种对比度差异值量化到图像特 征信息中则表现为统计两类图像中不同大小差异特 征幅值出现的次数, 即双模态红外图像中的差异特征 频次。
$K_{\mathrm{NN}}$ 概率密度估计 ${ }^{[10]}$ 是通过改变相同的小样本数 所需的区域大小来获得估计的概率密度序列值的。设 其所选定的区域内样本的个数为 $N$, 并根据总体样本 集的个数确定一个参数 $K_{N}$, 其中近邻数 $K_{N}$ 为正整数, $K_{N}$ 的大小决定了曲线的平滑程度 ${ }^{[11]}$, 当 $K_{N}$ 越大, 则 平滑程度也越大。 $K_{\mathrm{N} N}$ 近邻估计是在 $K_{N}$ 值固定的前提 下, 改变 $V$ 的大小进行概率密度估计, 可以更好地兼 顾在高密度区域估计得到的分辨率以及低密度区域 估计的连续性。

另一种概率密度估计方法为核密度估计法, 核密 度估计法是利用平滑的峰值函数拟合观察到的数据 点, 对真实的概率分布曲线进行模拟估计的一种非参 数估计法 ${ }^{[12]}$ 。但是核密度估计中涉及到内核函数的选 择, 常用的核密度估计函数有: 高斯核函数、 Epanechnikov 核函数、矩形核函数、三角形核函数、 伽马核函数以及余弦核函数等 ${ }^{[13]}$ 。同时核密度函数带 宽的不同对核密度估计的影响也很大，带宽反映了核 密度估计曲线的整体平滑程度, 同时反映了样本在整 体曲线分布中所占的比重。带宽越大, 则样本点在曲 线中所占比重越小, 整体曲线就越平坦; 反之则整体 曲线就越陡峭 ${ }^{[14]}$ 。目前对于自适应带宽的设定研究较 少, 通常所选的带宽是根据经验值来设定的, 采用固 定的经验值带宽的核密度估计产生的误差很大。所以 $K_{\mathrm{NN}}$ 概率密度估计是一种较为客观的非参数概率密度 估计方法。

\section{2 实验}

\section{1 源图像采集及预处理}

本实验所搭建的红外偏振探测系统由热红外成 像仪、检偏器、图像采集软件和主控平台组成。所采 用的长波红外热像仪 DM60 (型号) 是由浙江大立公 司生产, 工作波段范围为 $8 \sim 14 \mu \mathrm{m}$, 探测器焦平面的 像素为 $384 \times 288$, 视场角为 $16^{\circ} \times 12^{\circ}$, 而红外检偏器 选用美国爱特蒙特光学公司生产的 Zn-Se 全息线栅偏 振片, 有效光学口径为 $34 \mathrm{~mm}$, 消光比为 $100: 1$, 透 过率为 $90 \%$ 。由于本文选用 3 个偏振量的成像方式对 10 个不同的目标场景进行偏振图像采集, 并对各斯托 克斯参量 $(I 、 Q 、 U)$ 、偏振度、偏振角等图像信息解 算, 得到了相应的红外光强图像和解算后的偏振度图 像分别如图 1(a)、(b)所示, 并分别提取所需场景目标 的特征。利用 PCA 融合算法得到了源图像组的融合 图像, 如图 1(c)所示, 图像大小均为 $256 \times 256$, 并利 用 $16 \times 16$ 的不重叠窗口提取每个图像块的特征值。

\section{2 差异特征选取和融合有效度构造}

亮度特征、边缘特征、纹理特征属于双模态红 
外图像主要差异特征, 能够有效表征双模态红外图 像的互补性特征信息。灰度均值 (gray mean, M)、 边缘强度 (edge intensity, EI)、标准差 (standard deviation, $\mathrm{SD}$ )、平均梯度（average gradient, $A G$ ）分 别量化表示亮度特征、边缘特征中的边缘幅值强度、 反差度、边缘清晰度, 纹理特征选取 Tamura 纹理特 征中的粗粘度 (coarseness, CA)、对比度 (contrast, $\mathrm{CN}$ ) 来量化表示 ${ }^{[15]}$ 。通过表 1 计算得出源图像在 6 大主要差异特征下的幅值范围。利用融合有效度表示 一定融合算法下, 融合后的图像特征对融合前两类图 像的有效融合程度 ${ }^{[16]}$, 本文选取基于距离测度的余弦 相似性来表示融合有效度的大小 ${ }^{[4]}$, 如公式(8)所示:

$$
V=\frac{T_{\mathrm{F}} * \max \left(T_{\mathrm{I}}, T_{\mathrm{p}}\right)}{\sqrt{\left(T_{\mathrm{F}}\right)^{2}+\max \left(T_{\mathrm{I}}, T_{\mathrm{p}}\right)^{2}}}
$$

式中: $T_{\mathrm{F}}$ 表示融合图像的对应图像块特征值的强度。 $V \in(0,1]$, 其值越大, 融合有效度越好。

\section{差异特征属性只受到图像本身的影响, 与融合算}

法无关。选取第 4 组源图像进行说明, 构建差异特征 的基于 PCA 算法的融合有效度分布图, 如图 2 所示 为差异灰度均值和差异边缘强度的融合有效度分布 图。

\section{3 基于 $K_{\mathrm{NN}}$ 概率密度估计的差异特征频次分布构造} 通过上述方法得到每幅源图组基于 PCA 算法的 6 种差异特征幅值散点分布图, 为了更好地表示差异特 征幅值的连续性, 本文中将每种差异特征幅值点按从 小到大划分为 20 组, 即 $L=20$, 幅值区间组数设为 20 可以更好地提升幅值区分度并减少误差 ${ }^{[4]}$, 便于得 到差异特征幅值的不同频次值。利用累积分布函数求 出每种差异特征幅值的频率分布直方图, 在幅值频率 分布直方图中, 差异特征幅值出现频率为差异特征频 次值，统计得到的每个直方图面积即为每个幅值区间 内该差异特征频次的值。累积分布函数没有引入带宽 等外部概念, 不会丢失任何的数据信息, 通过累积分 布函数得到频率分布直方图是真实准确的 ${ }^{[17]}$ 。

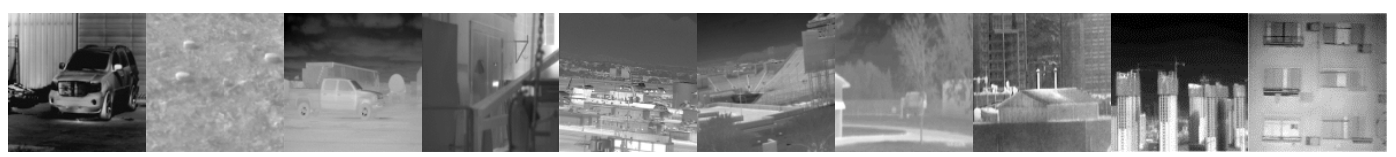

(a) 红外光强图像 (a) Infrared intensity images

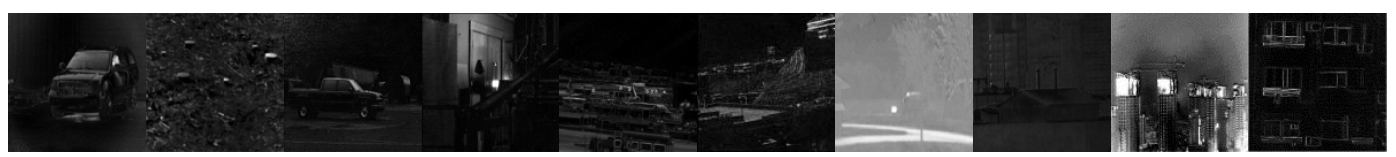

(b) 红外偏振度图像

(b) Infrared polarization images

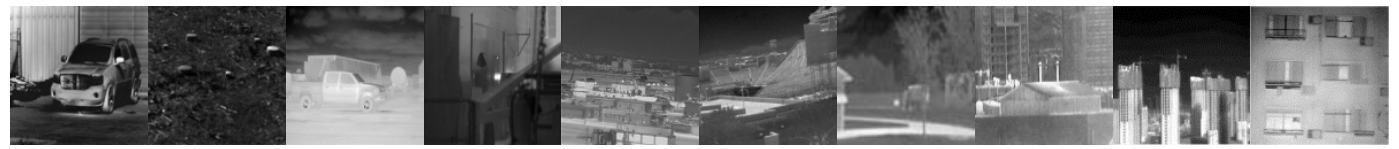

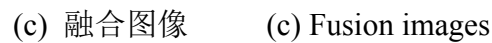

图 1 源双模态红外图像和融合图像 Fig.1 Source infrared intensity and infrared polarization images 表 1 源图像差异特征幅值范围 Table 1 Source images difference features ranges of amplitudes

\begin{tabular}{ccccccc}
\hline $\begin{array}{c}\text { Difference } \\
\text { features }\end{array}$ & M & EI & SD & AG & CA & CN \\
\hline I & {$[1.0820,174.9063]$} & {$[0.4587,244.4227]$} & {$[0.1197,59.7648]$} & {$[0.0041,2.3041]$} & {$[0,22.7222]$} & {$[0.0015,57.0951]$} \\
II & {$[90.2305,158.5078]$} & {$[0.0864,104.7186]$} & {$[0.0462,55.5542]$} & {$[0.0148,1.1381]$} & {$[0,21.7222]$} & {$[0.0041,48.6865]$} \\
III & {$[6.1367,184.4336]$} & {$[0.2973,83.7877]$} & {$[0.0170,37.7274]$} & {$[0.0018,1.4296]$} & {$[0,29.5000]$} & {$[0.0047,34.1911]$} \\
IV & {$[0.7656,227.7076]$} & {$[0.3037,130.7874]$} & {$[0.0142,46.2289]$} & {$[0.0001,1.6317]$} & {$[0,27.7778]$} & {$[0.0629,44.3780]$} \\
V & {$[33.7148,222.8984]$} & {$[0.0645,122.5487]$} & {$[0.0022,37.6050]$} & {$[0.0004,1.5880]$} & {$[0,19.4444]$} & {$[0.0155,37.0938]$} \\
VI & {$[10.9922,139.4141]$} & {$[0.0571,248.4669]$} & {$[0.1288,49.1117]$} & {$[0.0742,2.8657]$} & {$[0.1111,29.2778]$} & {$[0.0044,41.3021]$} \\
VII & {$[0.0234,84.0078]$} & {$[0.0010,53.0762]$} & {$[0.0273,30.5482]$} & {$[0.0002,0.5771]$} & {$[0,28.2778]$} & {$[0.0055,28.6887]$} \\
VIII & {$[27.5820,204.2969]$} & {$[13.0318,181.0189]$} & {$[2.5411,63.5971]$} & {$[0.2432,2.4438]$} & {$[0,17.7778]$} & {$[1.5427,62.5325]$} \\
IX & {$[0.7305,214.0742]$} & {$[0.0886,233.1556]$} & {$[0.0987,62.4078]$} & {$[0.7076,2.8640]$} & {$[0.0088,20.3860]$} & {$[0.0040,55.2712]$} \\
X & {$[31.6602,216.8047]$} & {$[0.1731,135.6868]$} & {$[0.0017,46.8076]$} & {$[0.0029,2.4309]$} & {$[0,22.4211]$} & {$[0.0049,29.0912]$} \\
\hline
\end{tabular}




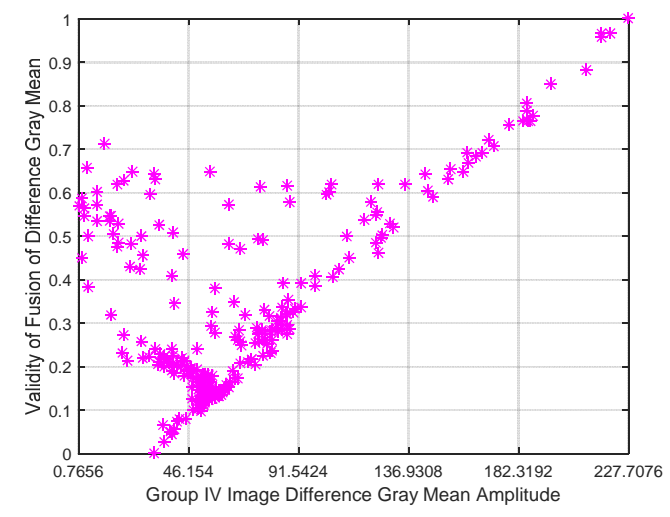

图 2 融合有效度分布图

Fig.

本文中利用基于 $K_{\mathrm{N} N}$ 概率密度估计的方法来构造 差异特征频次分布, 每幅源图组的每种差异特征幅值 有 256 个, 即 $N=256$, 选用 6 种差异特征, 原始样 本集 $\left\{T_{i}\right\}$ 所含样本个数 $N=256, i=1,2, \cdots, N$ 。原始样 本集里所含样本个数太少不足以满足非参数概率密 度估计所需样本数大的要求, 所以将差异特征幅值点 $T_{i}$ 移动的步长设为 $x_{\text {step }}=0.01$, 通过插值扩充了样本 集。扩充后的样本集 $\left\{T_{j}\right\}$ 为第 $a$ 种差异特征幅值样本 集, 其中 $a=1,2 \cdots, 6$, 所含样本个数为 $N^{\prime}=\left[T_{j \mathrm{r}}-T_{j 1} / x_{\text {step }}\right], j=1,2, \cdots, N^{\prime}$ 。 $T_{j 1}$ 为幅值差值样本 集的左边界, $T_{j \mathrm{r}}$ 为幅值差值样本集的右边界, 且每种 差异特征幅值样本集均服从同一种分布。当变量为幅 值点 $T_{j}$ 时, 通过调整包含 $T_{j}$ 区域的体积, 直到区域内 刚好落入 $K_{N}$ 个样本点, 所以 $K_{N}$ 取值不同时 $V$ 的值也 会随之改变, 这些样本被称为幅值样本点 $T_{j}$ 的 $K_{N}$ 个 最近邻。

本文的幅值样本点属于一维数据, 样本点相当 于分布在一条线上, 样本集 $\left\{T_{j}\right\}$ 中任取样本 $T_{j m}, V$ 的值等于幅值样本点 $T_{j m}$ 到它的第 $K_{N}$ 近邻距离 $T_{K_{N}(m)}$ 的两倍, 其中选用欧式距离衡量 $T_{j m}$ 与 $T_{K_{N}(m)}$ 间的距 离大小 ${ }^{[9]}$ 。随着步长的移动, 依次求出每一个幅值点 的概率密度估计值, 直到 $T_{j m}<T_{j \mathrm{r}}+x_{\mathrm{step}} / 2$ 。某一特定 的差异特征 $a$ 的幅值点 $T_{j m}$ 的概率密度估计值 $f\left(T_{j m}\right)$ 如下式所示:

$$
f\left(T_{j m}\right)=\frac{k_{N} / N^{\prime}}{2^{*}\left|T_{j m}-T_{K_{N}(m)}\right|}
$$

通常 $K_{N}=\sqrt{N^{\prime}}$, 若得到的数并非整数时, 则 $K_{N}$

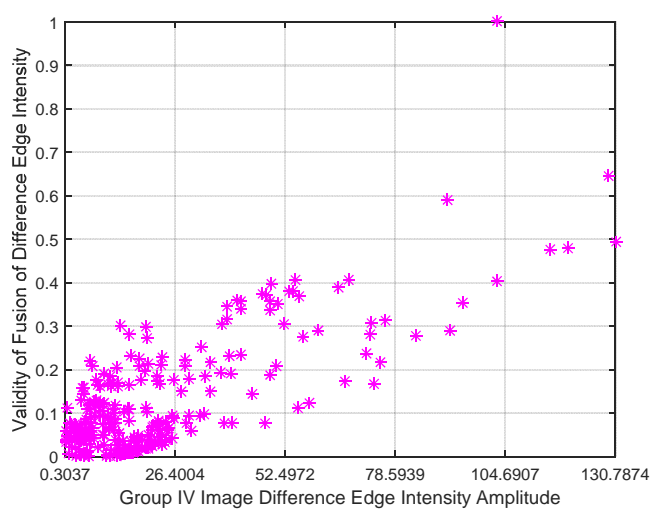

Distribution maps of fusion validity

向最靠近 0 的整数取整。如上式所示, 显然如果 $T_{j m}$ 幅值点附近的密度较高时, 体积 $V$ 则相对较小, 提升 了分辨力; 如果 $T_{j m}$ 幅值点附近的密度较低时, 体积 $V$ 则相对较大，进入高密度区会停止扩大。

通过 $K_{\mathrm{N} N}$ 概率密度估计得到的拟合曲线分布图中 曲线的纵坐标是差异特征幅值概率密度值与横坐标 对应差异特征幅值的比值, 概率密度估计曲线在所有 横坐标积分范围的面积恒为 1 。且在幅值概率密度估 计曲线中, 差异特征幅值密度为差异特征频次, 所以 通过计算概率密度估计分段曲线与横坐标子幅值区 间围成的面积求出的是差异特征幅值概率密度序列 值, 亦即差异特征频次概率序列值。概率密度拟合曲 线的表达式是未知的, 通过数值积分中的复化梯形积 分 ${ }^{[18]}$ 近似可以求得分段曲线与横坐标子区间围成的 面积。本文中将每组源图像的差异特征幅值区间 $\left[T_{j 1}, T_{j r}\right]$ 划分为 $n=20$ 个子区间，其中第 $k$ 个子区间 $\left[T_{j 1}, T_{j \mathrm{r}}\right]$ 也划分为 $n$ 份, 步长为 $h^{\prime}=\left(T_{r k}-T_{l k}\right) / n$, 每个 子区间包含 $q$ 个差异特征幅值概率密度估计值 $f\left(T_{j m}\right)$, 每个子区间的节点为 $T_{m k}=T_{1 k}+w h^{\prime}, w=$ $1,2, \cdots, n+1$, 在每个差异特征幅值子区间内使用复化 梯形积分, 近似求得每个差异特征幅值区间差异特征 频次的概率值，利用公式(10)表示:

$$
\begin{aligned}
f r_{k} & =256 * \int_{T_{l k}}^{T_{r k}}\left[\sum_{m=1}^{m=q} f\left(T_{j m}\right) \mathrm{d}\left(T_{j m}\right)\right] \mathrm{d}\left(T_{j}\right) \\
& =256 * \frac{h^{\prime}}{2}\left[f\left(T_{l k}\right)+2 \sum_{w=1}^{n-1} f\left(T_{l k}+w h^{\prime}\right)+f\left(T_{r k}\right)\right]
\end{aligned}
$$

通过上式得出了每组源图像每种差异特征下基 于 $K_{\mathrm{N} N}$ 概率密度估计的差异特征频次具有统计意义的 序列值 $\left\{f r_{k}\right\}$, 从而构造出差异特征频次分布。

\section{3 实验结果与分析}

\section{1 主观分析}

本文采用 Matlab 2014a 作为实验软件平台, 实验 
运行环境为 $3 \mathrm{GHz}$ Intel Core i7 PC 机, 对所选取的 10 组双模态红外图像采用同一种融合算法进行融合 (以 PCA 算法为例)。选用 Rosenblatt 提出的积分均方误 差 (integral mean square error, MISE) 修正固定核密度 带宽参数确定最优带宽 ${ }^{[19]}$ 的高斯核密度估计与本文

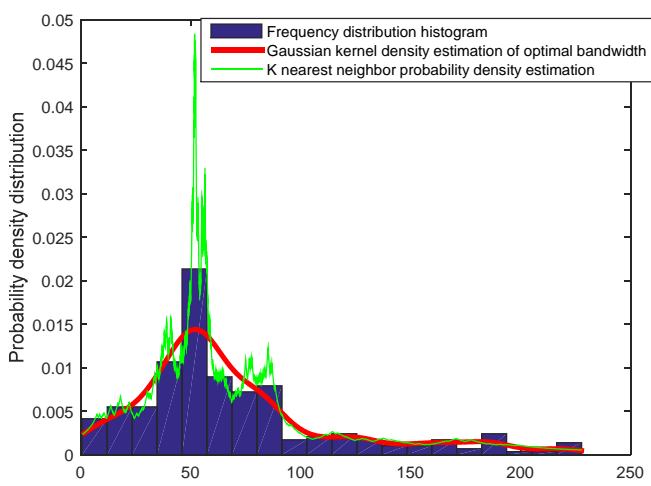

(a) 差异灰度均值幅值概率密度分布图

(a) Difference gray mean amplitude probability density maps

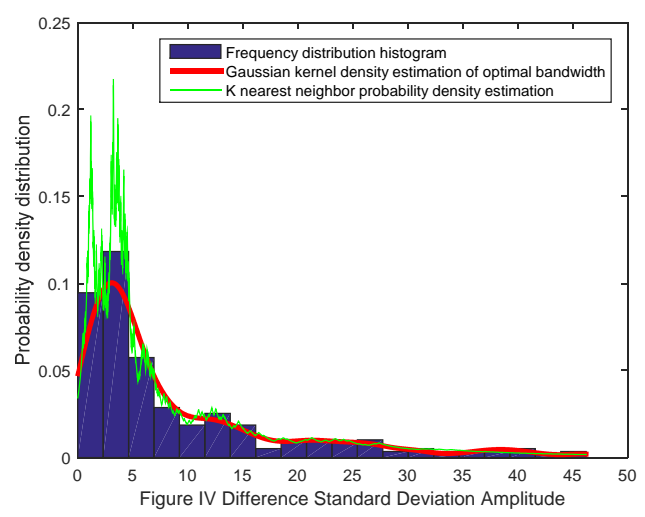

(c) 差异标准差幅值概率密度分布图

(c) Difference standard deviation amplitude probability density maps

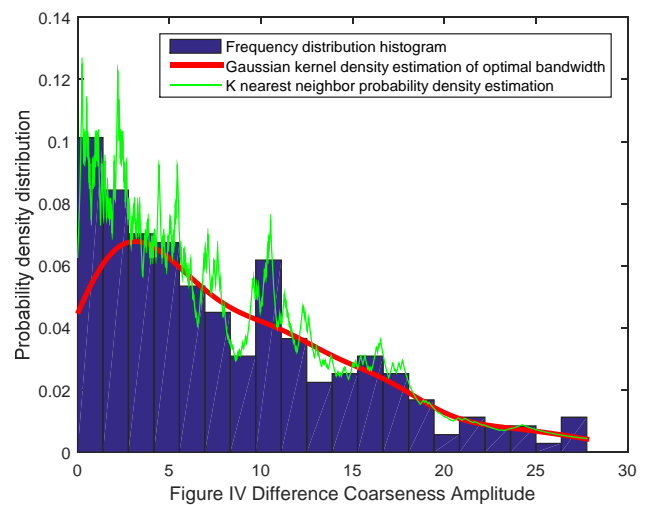

(e) 差异粗粘度幅值概率密度分布图

(e) Difference coarseness amplitude probability density maps

图 3 差异特征幅值概率密度分布图
方法进行对比实验, 将累积分布函数统计得到的差异 特征幅值的频率分布直方图、MISE 高斯核密度估计 以及本文方法得到的差异特征幅值概率密度曲线进 行对比, 以图 “IV” 的 6 种差异特征为例, 如图 3 所 示。

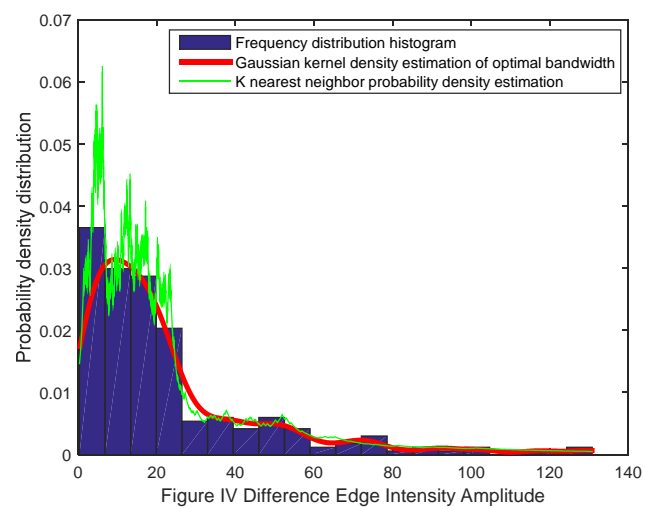

(b) 差异边缘强度幅值概率密度分布图

(b) Difference edge intensity amplitude probability density maps

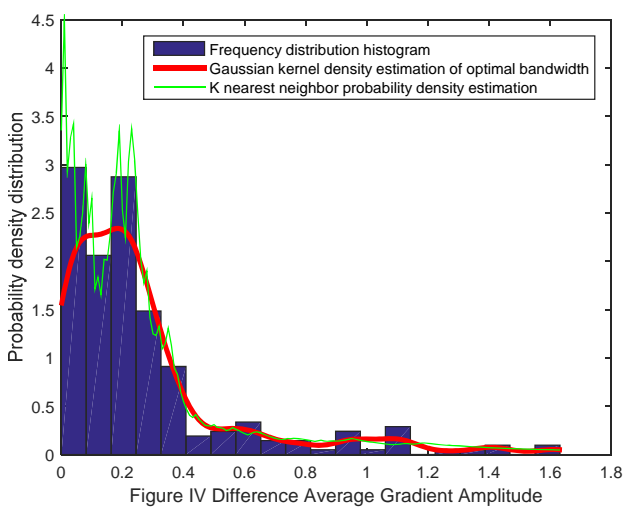

（d）差异平均梯度幅值概率密度分布图

(d) Difference average gradient amplitude probability density maps

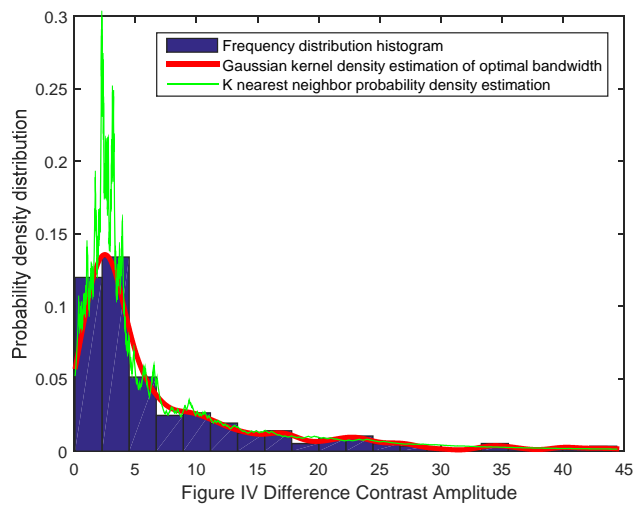

(f) 差异对比度幅值概率密度分布图

(f) Difference contrast amplitude probability density maps

Fig.3 Difference feature amplitude probability density maps 
利用复化梯形积分构造出本文方法与最优带宽 高斯核密度估计的差异特征频次分布, 同时利用累积 分布函数得到差异特征频次分布, 每组实验源图像均 分为 20 个幅值子区间, 不同实验源图像的差异特征 频次分布用虚线隔开，如图 4 所示。

从图 3 中可以看出, 本文方法的差异特征幅值概 率密度曲线包络与差异特征幅值的频率分布直方图 更加接近 (图 3 中以第 4 组双模态红外图像为例进行

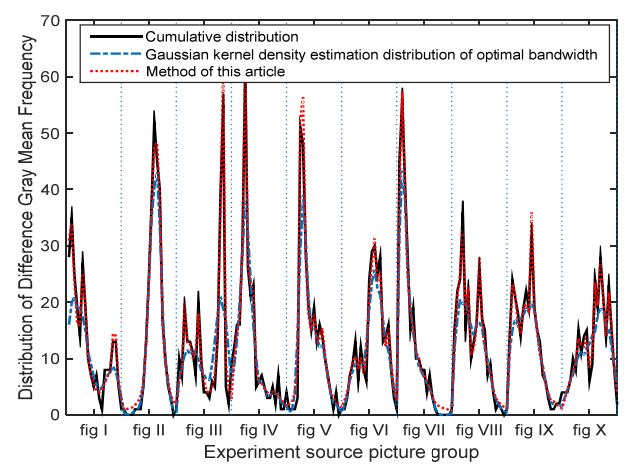

(a) 差异灰度均值频次分布图

(a) Distribution maps of difference gray mean frequency

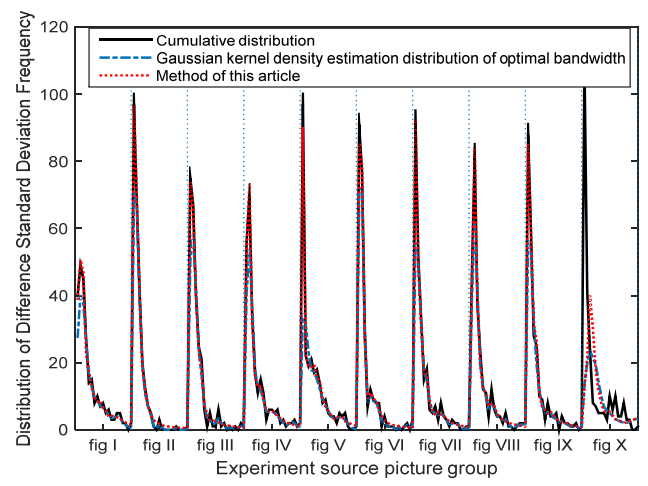

(c) 差异标准差频次分布图

(c) Distribution maps of difference standard frequency

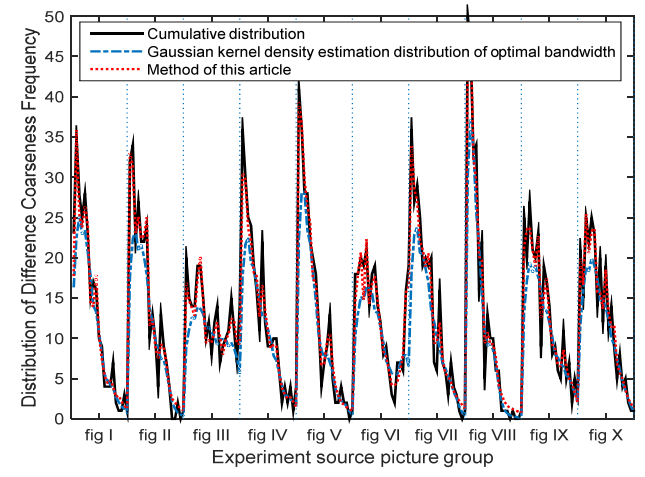

(e) 差异粗糙度频次分布图

(e) Distribution maps of difference coarseness frequency
说明, 其他源图像组实验结果与第 4 组源图像相同)。 图 4 中本文方法所构造的差异特征频次分布曲线与累 积分布函数得到的真实分布曲线在 6 种差异特征频次 分布构造中重合程度很高, 而基于 MISE 的最优带宽 高斯核密度估计所构造的差异特征频次分布曲线与 累积分布相比有一些偏差，尤其在差异灰度均值以及 差异粗粘度频次分布构造中偏离程度较大。显然本文 所提的方法构造差异特征频次分布更加准确。

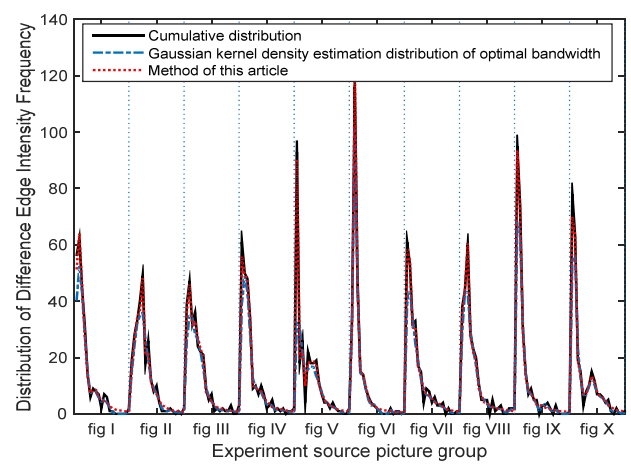

(b) 差异边缘强度频次分布图

(b) Distribution maps of difference edge intensity frequency

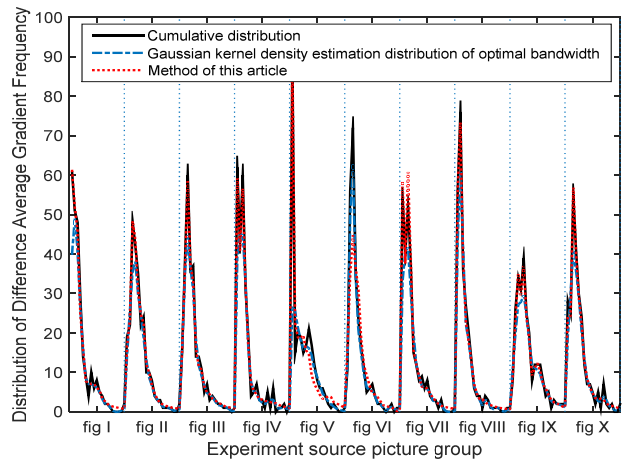

(d) 差异平均梯度频次分布图

(d) Distribution maps of difference average gradient frequency

图 4 差异特征频次分布图 Fig.4 Distribution maps of difference feature frequency 
相比较本文所提方法, MISE 最优带宽高斯核密 度估计构造差异特征频次分布时产生的偏差较大, 是 因为如下原因:

1) 选取核函数内核时, 鉴于核函数在波形合成 计算上的易用性, 选择了高斯内核作为核密度估计函 数 ${ }^{[20]}$;

2) 采用 MISE 最小化误差函数确定核密度函数 最优带宽 $h$ 时, 是求差异特征幅值样本集的高斯核密 度估计得到的概率密度函数 $\hat{f}_{h}\left(T_{j m}\right)$ 与原始幅值样本 集的概率密度函数 $f\left(T_{i m}\right)$ 差值的均值, 并作积分, 进 而求解最小化问题。原始差异特征幅值样本集的概率 密度函数 $f\left(T_{i m}\right)$ 是未知的, 所以将 Sliverman 提出的经 验法则法 (rule-of-thumb, ROT) 用于核密度函数带宽 的选择 ${ }^{[20]}$, 即假设 $f\left(T_{i m}\right)$ 是基于高斯核方差为 $\sigma^{2}$ 的正 态分布族。

正是上述内核函数与带宽确定造成的双重误差, 导致 MISE 最优带宽高斯核密度估计在差异特征频次 构造中的不足，也体现了本文方法的准确性。

\section{2 客观分析}

分别计算最优带宽高斯核密度估计 (方法 1) 以及 本文方法 (方法 2)这两种频次构造方法与累积分布 (真

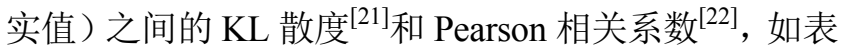
2 和表 3 所示。在表 2 中, 在 6 种差异特征中, 本文 方法相较于 MISE 最优带宽高斯核密度估计的 KL 散 度较小, 且差异灰度均值 (M) 和差异粗䊚度 (CA) 二者散度差最大, 差异标准差 (SD) 散度差小, 亦即 本文算法所构造的差异特征频次分布与该差异特征 频次的累积分布相关性大, 尤其在 M 和 CA 中的相关 性相较于最优带宽高斯核密度估计更大。

在表 3 中, 本文方法的 Pearson 相关系数比最优 带宽高斯核密度估计得到的结果高, 二者 Pearson 相 关系数的差值在 $\mathrm{M}$ 和 $\mathrm{CA}$ 中最大, 其他 4 类差异特征 的 Pearson 相关系数差值较小, 本文方法与最优带宽 高斯核密度估计的线性相关程度均为极强相关, 但是 本文算法的线性相关程度比最优带宽高斯核密度估 计更高。综上, 通过本文算法构造出的差异特征频次 分布能够准确地反映真实的差异特征频次分布, 且相 较于另一种构造方法误差较小。

\section{4 结论}

本文通过构造 10 组双模态红外图像基于 $K_{\mathrm{NN}}$ 概 率密度估计的差异特征频次分布, 并求解本文构造方 法的频次分布和 MISE 最优带宽高斯核密度估计的频 次分布分别与累积分布的相似性测度, 得出如下结论:
1）非参数概率密度估计法应用于差异特征频次分布 构造中具有可行性；2）从对比实验中得出本文方法 对于差异特征频次分布构造的准确性优于 MISE 最优 带宽高斯核密度估计；3）为下一步研究基于双模态 红外图像差异特征多属性的融合有效度分布合成奠 定基础。

表 2 不同频次构造方法与真实值的 KL 散度比较

Table 2 Comparison of KL divergence between different frequency construction methods and real values

\begin{tabular}{ccc}
\hline Difference features & Method 1 & Method 2 \\
\hline M & 0.0596 & 0.0243 \\
EI & 0.0429 & 0.0312 \\
SD & 0.1017 & 0.1011 \\
AG & 0.0402 & 0.0323 \\
CA & 0.0454 & 0.0192 \\
CN & 0.0327 & 0.0267 \\
\hline
\end{tabular}

表 3 不同频次构造方法与真实值的 Pearson 相关系数比较

Table 3 Comparison of Pearson correlation coefficients between different frequency construction methods and real values

\begin{tabular}{ccc}
\hline Difference features & Method 1 & Method 2 \\
\hline M & 0.9129 & 0.9873 \\
EI & 0.9622 & 0.9962 \\
SD & 0.9086 & 0.9363 \\
AG & 0.9503 & 0.9830 \\
CA & 0.9177 & 0.9822 \\
CN & 0.9705 & 0.9978 \\
\hline
\end{tabular}

\section{参考文献:}

[1] 安富, 杨风暴, 牛涛. 模糊逻辑与特征差异驱动的红外偏振图像融合 模型 $[\mathrm{J}$. 红外技术, 2014, 36(4): 304-310.

AN Fu, YANG Fengbao, NIU Tao. A fusion model of infrared polarization images based on fuzzy logic and feature difference driving[J]. Infrared Technology, 2014, 36(4): 304-310.

[2] 牛涛, 杨风暴, 王肖霞, 等. 差异特征与融合算法的集值映射关系的 建立[J]. 红外与激光工程, 2015, 44(3): 1073-1079.

NIU Tao, YANG Fengbao, WANG Xiaoxia, et al. Establishment of set -valued mapping between difference characteristics and fusion algorithms[J]. Infrared and Laser Engineering, 2015, 44(3): 1073-1079.

[3] 牛涛, 杨风暴, 王志社, 等. 一种双模态红外图像的集值映射融合方 法[J]. 光电工程, 2015, 42(4): 75-80.

NIU Tao, YANG Fengbao, WANG Zhishe, et al. A set-valued mapping fusion method of dual-mode infrared image[J]. Opto-Electronic Engineering, 2015, 42(4): 75-80.

[4] 郭吉. 双模态红外图像融合有效度分布的合成研究[D]. 太原: 中北大 
学, 2018.

GUO Zhe. Study on Synthesis of Fusion Validity Distribution of Bimodal Infrared Images[D]. Taiyuan: North University of China, 2018.

[5] 张玉敏. 基于不同核函数的概率密度函数估计比较研究 [D]. 石家庄: 河北大学, 2010 .

ZHANG Yumin. The Comparative Study of Probability Density Function Estimation Based on the Different Kernel Functions[D]. Shijiazhuang: Hebei University, 2010.

[6] 牛继勇, 李范鸣, 马利祥. 目标红外偏振探测原理及特性分析 [J]. 红 外技术, 2014, 36(3): 215-220.

NIU Jiyong, LI Fanming, MA Lixiang. The principle and characteristics analysis of IR polarization detection[J]. Infrared Technology, 2014, 36(3): 215-220.

[7] 陈添丁, 王娃, 朱敬意, 等. 基于图像偏振特性的野外环境积水区域 检测 $[\mathrm{J}]$. 光电工程, 2013(4): 14-23.

CHEN Tianding, WANG Wa, ZHOU Zhengyin. Wild environment water hazards detection based on polarization information of image[J]. Opto-Electronic Engineering, 2013(4): 14-23.

[8] 虞文俊. 红外偏振成像技术与方法研究[D]. 南京: 南京理工大学, 2014.

YU Wenjun. Research on Infrared Polarization Imaging Technology and Method[D]. Nanjing: Nanjing University of Science and Technology, 2014.

[9] 韩平丽. 红外辐射偏振特性及目标识别研究[D]. 西安: 西安电子科技 大学, 2014 .

HAN Pingli. Research in Target Recognition Employing Polarization Signatures of Thermal Emission[D]. Xi'an: Xidian University, 2014.

[10] Mack Y P, MackM Rosenblatt. Multivariatek-nearest neighbor density estimates[J]. Journal of Multivariate Analysis, 1979(9): 1-15.

[11] Hall P, Park B U, Samworth R J. Choice of neighbor order in nearest -neighbor classification[J]. The Annals of Statistics, 2008, 3(6): 2135-2152.

[12] PARZEN E. On estimation of a probability density function and mode[J]. The Annals of Mathematical Statistics, 1962, 33(3): 1065-1076.

[13] 王振武, 何关瑶. 核函数选择方法研究 $[\mathrm{J}]$. 湖南大学学报: 自然科学 版, 2018, 45(10): 155-160.

WANG Zhenwu, HE Guanyao. Research on selection method of Kernel function[J]. Journal of Hunan University: Natural Sciences, 2018, 45(10): 155-160

[14] 黎运发, 黄名辉. 核密度估计逐点最优窗宽选择的改进 $[\mathrm{J}]$. 统计与决 策, 2011(14): 28-32.
LI Yunfa, HUANG Minghui. Improvement of point-by-point optimal window width selection for kernel density estimation[J]. Statistics \& Decision, 2011(14): 28-32.

[15] Tamura Hideyuki, Mori Shunji, Yamawaki Takashi. Textural features corresponding tovisual perception[J]. IEEE Transactions on Systems, Man and Cybernetics, 1978, 8(6): 460-473.

[16] 杨风暴, 李伟伟, 萄素珍, 等. 红外偏振与红外光强图像的融合研究 [J]. 红外技术, 2011, 33(5): 262-266.

YANG Fengbao, LI Weiwei, LIN Suzhen, et al. Study on fusion of infrared polarization and intensity images[J]. Infrared Technology, 2011, 33(5): 262-266.

[17] 陈蒙. 一种基于累积分布函数变换法的直方图均衡化图像增强应用 研究[J]. 贵州大学学报: 自然科学版, 2013, 30(6): 107-109, 137.

CHEN Meng. Study on the stability of surrounding rock in soft rock tunnel with large-span[J]. Journal of Guizhou University: Natural Sciences, 2013, 30(6): 107-109, 137.

[18] 邢诚, 王建强, 贾志强. 多种数值积分方法比较分析 [J]. 城市勘测, 2010(1): 104-106.

XING Cheng, WANG Jianqiang, JIA Zhiqiang. Comparative analysis of various numerical integration methods[J]. Urban Geotechnical Investigation \& Surveying, 2010(1): 104-106.

[19] 马梦知, 范厚明, 黄莒森, 等. 基于非参数核密度估计的集装箱码头 交通需求预测模型 $[J]$. 大连海事大学学报, 2019, 45(1): 74-81.

MA Mengzhi, FAN Houming, HUANG Jusen, et al. Traffic demand forecasting model for container terminal based on non-parametric kernel density estimation[J]. Journal of Dalian Maritime University, 2019, 45(1): 74-81.

[20] 徐玉琴, 张扬, 戴志辉. 基于非参数核密度估计和 Copula 函数的配 电网供电可靠性预测 [J]. 华北电力大学学报, 2017, 44(6): 14-19.

XU Yuqin, ZHANG Yang, DAI Zhihui. Power supply reliability prediction of distribution network based on nonparametric kernel density estimation and copula function[J]. Journal of North China Electric Power University, 2017, 44(6): 14-19.

[21] Kullback S, Leibler R A. On information and sufficiency[J]. The Annals of Mathematical Statistics,1951(22): 79-86.

[22] 李鸿林, 张琦, 杨大伟. 无参考模糊图像质量评价改进算法[J]. 计算 机应用, 2014, 34(3): 797-800.

LI Honglin, ZHANG Qi, YANG Dawei. Improved algorithm for no-reference quality assessment of blurred image[J]. Journal of Computer Applications, 2014, 34(3): 797-800. 